\title{
Aspects of pre-excitation and its elucidation by His bundle electrograms
}

\author{
Charles S. Smithen ${ }^{1}$ and Dennis M. Krikler \\ From the Departments of Cardiology, Guy's Hospital, and The Prince of Wales's Hospital, \\ London
}

His bundle electrograms were performed in 4 patients illustrating the varied aspects of the preexcitation syndrome. In 3 cases of Wolff-Parkinson-White syndrome the $Q R S$ complexes were found to be formed by fusion between the normal and premature activation processes. In one of these cases a re-entry tachycardia was shown, with antegrade conduction occurring through the His bundle and retrograde conduction bypassing it. These observations are consistent with the hypothesis that an anatomical bypass of the normal atrioventricular conduction system is responsible for Wolff-Parkinson-White syndrome. One case of Lown-Ganong-Levine syndrome with recurrent atrial flutter was found to have a reduced $A$-His interval representing the time from low right atrial to His bundle activation. This finding is compatible with a rapidly conducting atrioventricular nodal bypass. The implications of these observations are discussed.

The unduly rapid conduction of the cardiac impulse along preferential pathways may produce unusual electrocardiographic appearances, and this may be associated with a tendency for supraventricular arrhythmias to occur. Whereas previously the Wolff-Parkinson-White syndrome, the characteristic form of pre-excitation, was considered to be entirely benign, it is now recognized that complicating arrhythmias may be difficult to prevent or treat and that they are potentially lethal.

While in the classical case the syndrome is easily recognized on the conventional electrocardiogram, difficulties may arise in variant forms. The spectrum includes: (I) the classical Wolff-Parkinson-White syndrome with a short PR and a wide QRS showing initial slurring due to a delta wave (type $A$ where there is a dominant $R$ in $V I$; type $B$, when the main QRS deflection in VI is downwards, according to the definition of Rosenbaum et al., 1945); (2) atypical Wolff-Parkinson-White syndromes with normal PR and abnormal QRS complexes; or short PR, delta wave, and narrow QRS; and (3) the syndrome of short $P R$, normal QRS, and no delta wave (Lown, Ganong, and Levine, 1952).

Received 30 September 1971.

1 Present address: The New York Hospital - Cornell Medical Center, New York.
Recent experimental and pathological evidence has supported the view that an anatomical bypass of the normal atrioventricular conduction system is responsible for the pathogenesis of this entity (Durrer, Schuilenburg, and Wellens, 1970; Lev et al., 1966). We have utilized the technique of recording His bundle electrograms to investigate 4 patients who illustrate the varied aspects of the pre-excitation syndrome.

\section{Methods}

All patients were studied in the supine position at rest without premedication, and informed consent was obtained in each case. The His bundle electrogram was recorded via a bipolar electrode catheter $^{1}$ introduced into the femoral vein and positioned across the tricuspid ring under fluoroscopic control as described previously (Scherlag et al., 1969; Smithen and Sowton, 1971; Smithen et al., 1972). His bundle potentials were recorded over a frequency band of 40500 cps (HBE lead); the catheter position was adjusted until a sharp biphasic deflection appeared between the $P$ wave and QRS complex of a standard lead electrocardiogram recorded simultaneously. Records were taken on a Cambridge six-channel photographic recorder with a paper speed of $100 \mathrm{~mm} / \mathrm{sec}$.

A second bipolar electrode catheter was introduced through an arm vein, advanced to the junction of the right atrium and superior vena

1 United States Catheter Inc., Glens Falls, New York. 
FIG. I Electrocardiogram of Case I showing incomplete pattern of type $A$ Wolff-ParkinsonWhite syndrome, and possible anterolateral cardiac infarction, recorded in 1970.

FIG. 2 Rhythm strips (lead III), Case I. (a) Atrial fibrillation with an irregular ventricular response of approximately $160 / \mathrm{sec}$ : the Wolff-Parkinson-White pattern is maintained with wide $Q R S$ complexes (delta waves). (b) Paroxysmal supraventricular tachycardia at 200/min with narrow $Q R S$ complexes and no delta waves. The ST segments are depressed I $\mathrm{mm}$.

FIG. 3 His bundle electrogram of Case I during sinus rhythm when there was little evidence of pre-excitation. The P-His interval is $90 \mathrm{msec}$ and the His- $Q$ interval is $50 \mathrm{msec}$. $A s$ in all Figures, $H B E=H i$ bundle electrogram; $A=$ atrial activity; $V=$ ventricular activity; $H=$ His deflection.

FIG. 4 His bundle electrogram of Case I during atrial pacing at progressively shorter cycle lengths. The P-His interval progressively increases but there is a progressive reduction in the His- $Q$ interval. At a cycle length of $570 \mathrm{msec}$ the His $-Q=40 \mathrm{msec}$, at $400 \mathrm{msec}$ the His- $Q=15$ msec, and at $355 \mathrm{msec}$ the His $-Q=0$, indicating simultaneous activation of the His bundle and the ventricle. $S T=$ pacing stimulus.

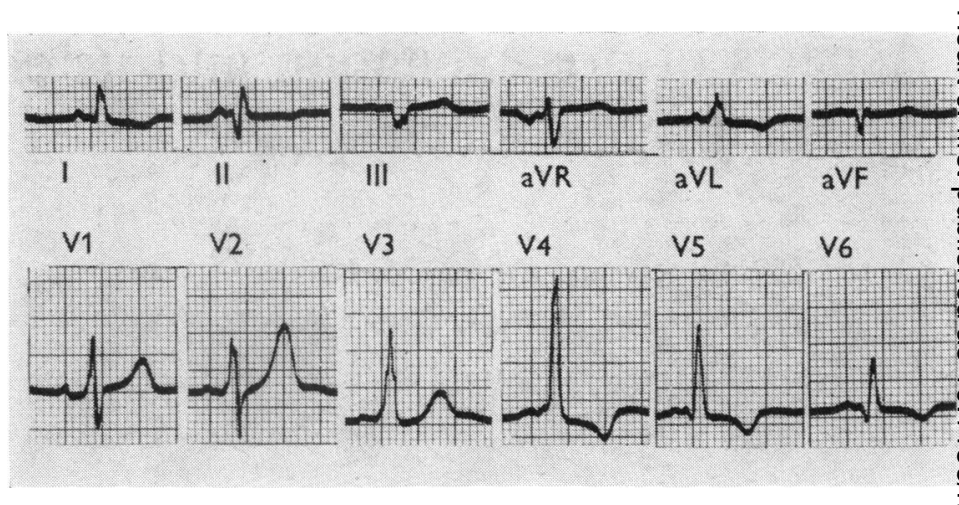



a
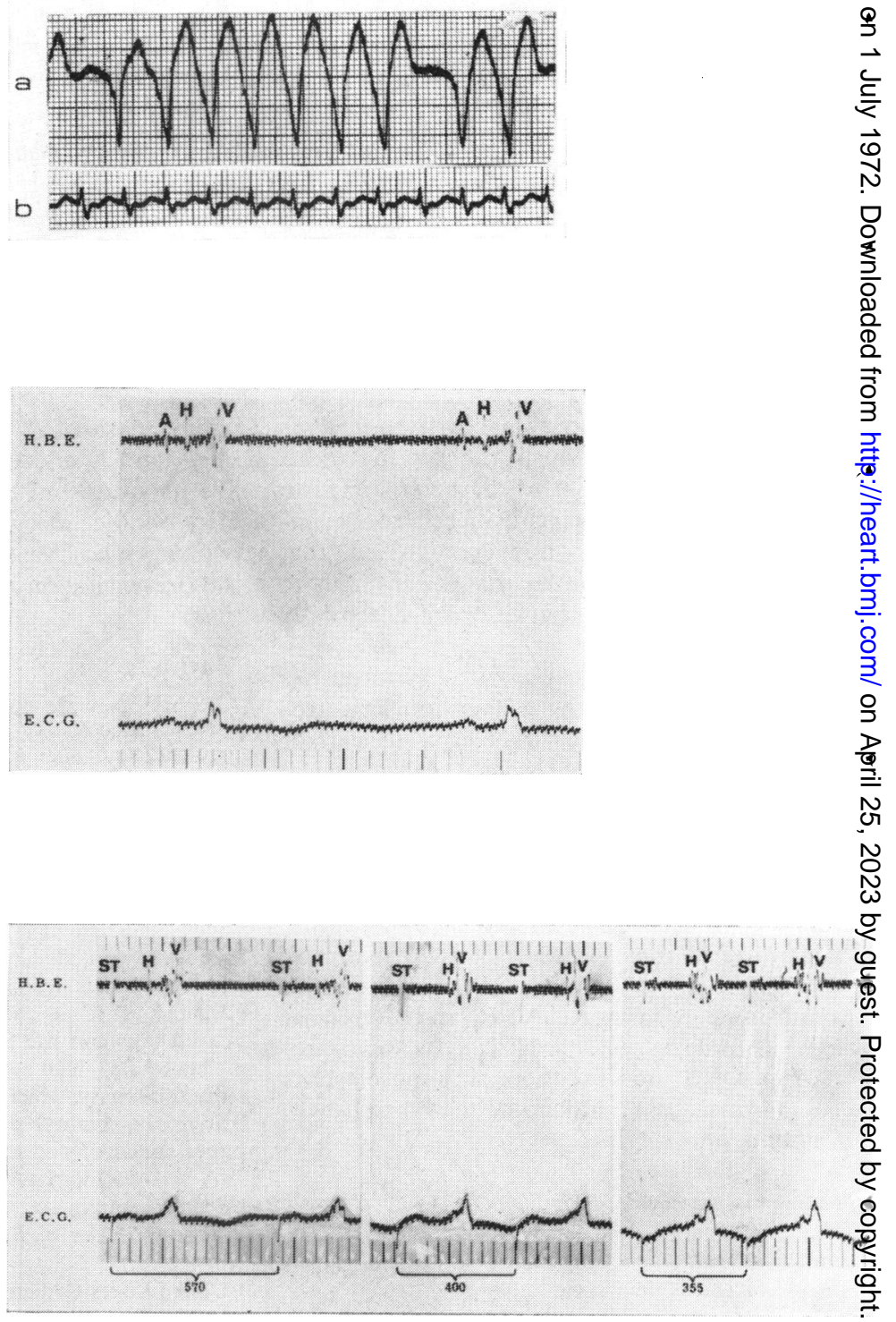
cava, and used to pace the atrium with an external battery-powered pacemaker.

The following measurements were made. The PR interval, representing intra-atrial, atrioventricular nodal, and intraventricular conduction times, was measured as in conventional electrocardiography. The P-His interval, representing atrial and atrioventricular nodal conduction, was measured from the beginning of the $P$ wave or pacing stimulus to the beginning of the His potential. The His-Q or His-V interval, representing intraventricular conduction, was measured from the His potential to the onset of ventricular depolarization in the standard lead. The A-His interval, representing the time between low right atrial and $\mathrm{His}$ bundle activation, was measured in one patient from the onset of the atrial potential in the HBE lead to the His deflection. The normal values in sinus rhythm, obtained previously on ro patients without AV conduction abnormalities, agreed well with those established in the published reports; delays of 80-140 msec for the P-His interval, and $35-55 \mathrm{msec}$ for the His-Q interval were used (Smithen and Sowton, I97I; Smithen et al., I972).

\section{Cases studied}

Case I A 65-year-old man developed stabbing left chest pain at the age of 56. Later an electrocardiogram showed the appearances of type $A$ Wolff-Parkinson-White syndrome (Fig. I). In the past year he had had frequent attacks of palpitations, associated with faintness, crushing central chest pain, sweating, and dyspnoea. With the first attack he was suspected of having a myocardial infarction and was admitted to hospital: the electrocardiogram showed atrial fibrillation with persistent features of the Wolff-Parkinson-White syndrome (Fig. 2a). Subsequent attacks have been less severe and have usually shown paroxysmal supraventricular tachycardia, with narrow QRS complexes (Fig. 2b), with more conspicuous ST segment depression in the chest leads; occasionally atrial fibrillation has recurred. In tracings taken between attacks there have been, in addition to the Wolff-Parkinson-White pattern, fairly numerous ventricular extrasystoles. The attacks were not prevented by procainamide or practolol but were successfully suppressed by quinidine.

FIG. 7 His bundle electrogram of Case 2 during atrial pacing with progressively shorter lengths. The P-His interval progressively increases but there is a progressive reduction in the His- $Q$ interval. At a cycle length of $515 \mathrm{msec}$ the His- $Q$ interval is $30 \mathrm{msec}$, at $440 \mathrm{msec}$ the His- $Q$ interval is $25 \mathrm{msec}$, and at 390 msec there is simultaneous activation of the His bundle and ventricle, with a change in the $Q R S$ configuration.

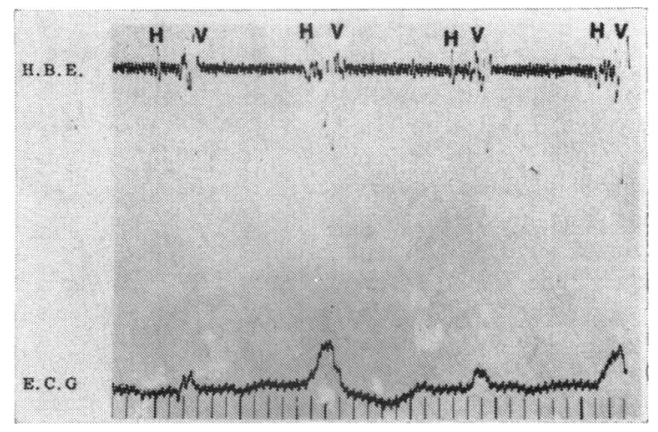

FIG. 5 His bundle electrogram of Case I during sinus rhythm with varying degrees of pre-excitation. Though no obvious $P$ waves are visible on the electrocardiogram the $H-H$ interval is regular. The $H-Q$ interval is 50 msec for the relatively normal complexes (Ist and $3 r d)$, and is o for the complexes showing pre-excitation (2nd and 4th). Spontaneous increases in the degree of pre-excitation are therefore associated with gross shortening of the His- $Q$ interval.

FIG. 6 His bundle electrogram of Case 2 during sinus rhythm and minimal evidence of pre-excitation. The P-His interval is $80 \mathrm{msec}$ and the His-Q interval is $40 \mathrm{msec}$.
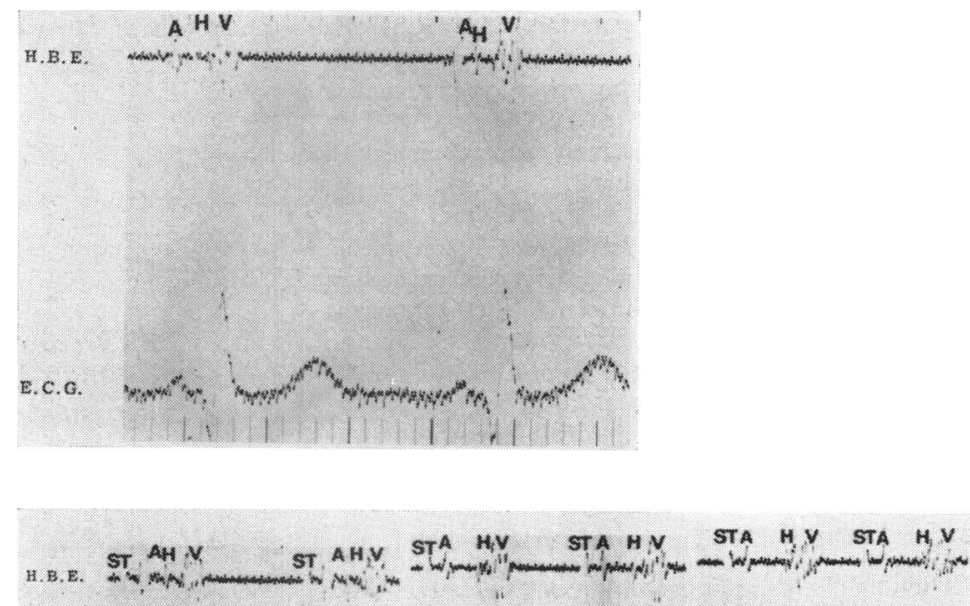


\section{Electrophysiological study}

In sinus rhythm, at a time when there was little evidence of pre-excitation, His bundle potentials were clearly seen, with normal $P$-His and His-Q intervals (Fig. 3). As the cycle length was progressively decreased by atrial pacing, pre-excitation became more evident (Fig. 4). The expected increase in the P-His interval occurred but there was progressive reduction in the His- $Q$ interval. At a cycle length of $355 \mathrm{msec}$ the His- $Q$ interval was $O$, indicating simultaneous activation of the His bundle and the ventricle and thus implying that the latter was depolarized early. During sinus rhythm, increases in the degree of preexcitation were shown by gross shortening of the His-Q interval to $O$ (Fig. 5).

Case 2 This 74-year-old man had suffered from paroxysmal tachycardia for 54 years. Fourteen years before the present admission, when the attacks became worse, he was found to have the Wolff-Parkinson-White syndrome type A. The attacks are partially controlled by practolol. There are no other clinical abnormalities apart from mild emphysema.

\section{Electrophysiological study}

Fig. 6 shows normal P-His and His-Q intervals during sinus rhythm when there was minimal evidence of pre-excitation. Atrial pacing with progressive reduction in the cycle length results in the expected P-His prolongation (Fig. 7); the His-Q interval progressively decreases and at a cycle length of $390 \mathrm{msec}$ there is simultaneous activation of the His bundle and ventricle, with a change in the QRS configuration. Unfortunately, the electrocardiographic tracing does not permit accurate assessment of the onset of ventricular activation, but the His-V interval as measured on the HBE lead progressively decreases with shortening of the cycle length.

During right ventricular stimulation, the patient developed supraventricular tachycardia (Fig. 8). A His potential is clearly seen preceding each QRS complex with a normal His-Q interval; there are no retrograde His potentials. Though we were unable to study the beginning and end of the tachycardia the appearances are consistent with a re-entry mechanism with antegrade conduction by the bundle of $\mathrm{His}$ and retrograde conduction via an anatomical tract bypassing the bundle of His.

Case 3 This 49-year-old man had been seen first 18 months previously with mild left mammary pain, and was found to have intermittent Wolff-Parkinson-White complexes, type B, alternating with normal beats (Fig. 9). He has never experienced an arrhythmia and is well in every other respect.

\section{Electrophysiological study}

The His bundle recordings during sinus rhythm at a cycle length of $850 \mathrm{msec}$ and during atrial pacing at cycle lengths of $620 \mathrm{msec}$ and $440 \mathrm{msec}$

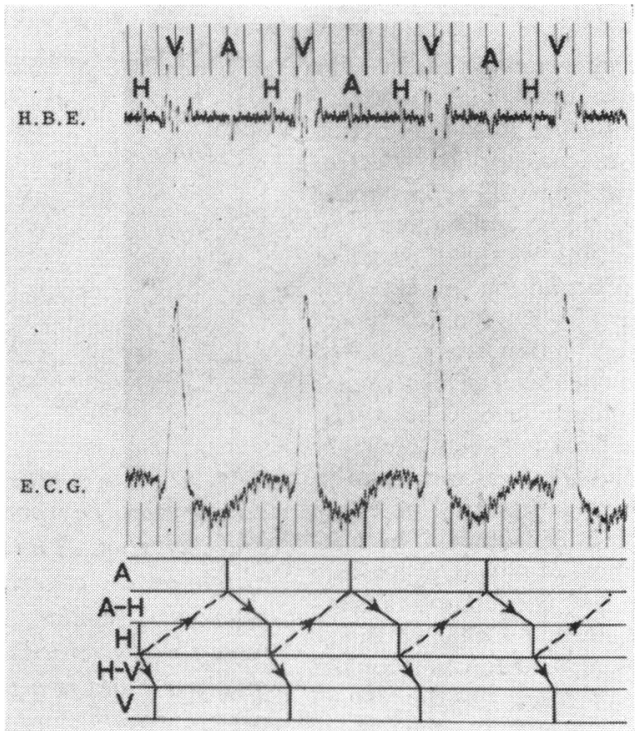

FIG. 8 His bundle electrogram of Case 2 during supraventricular tachycardia induced by right ventricular stimulation. $A$ His potential is clearly seen preceding each $Q R S$ complex with a His- $Q$ interval of $40 \mathrm{msec}$. No retrograde His potentials are seen. The appearance of the tachycardia is consistent with a re-entry mechanism with antegrade conduction by the bundle of His and retrograde conduction via an anatomical tract bypassing the bundle of His (dotted line).

FIG. 9 Electrocardiogram of Case 3 showing intermittent Wolf-Parkinson-White syndrome type $B$, alternating with normal beats.

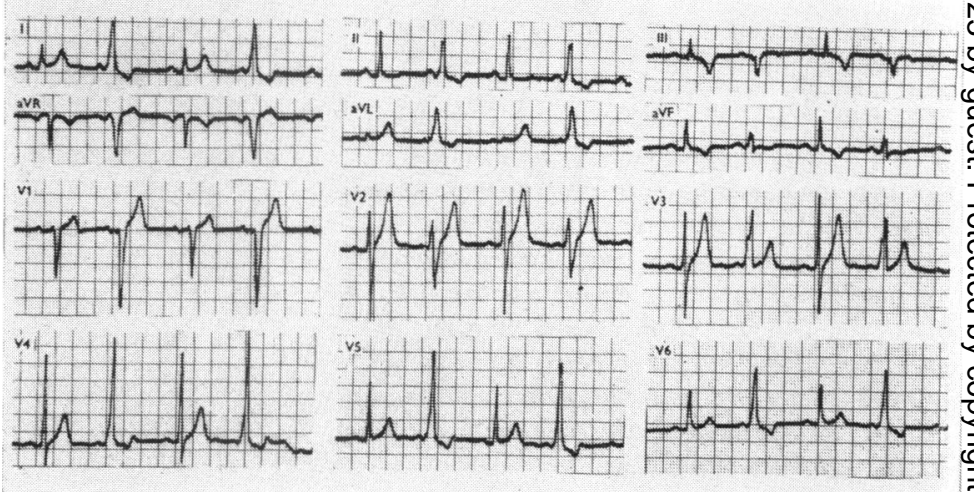


are shown in Fig. 10. A consistent pre-excitation pattern is evident during sinus rhythm and does not significantly change during atrial pacing. The His- $Q$ interval is reduced to $15 \mathrm{msec}$ during sinus rhythm and remains unchanged at the faster pacing rates. It was impossible to produce any arrhythmias by atrial or ventricular stimulation.

Case 4 This 62-year-old man was found collapsed at home. There were no cardiovascular abnormalities clinically. The features of tabes dorsalis were present and serological tests for syphilis were positive. An electrocardiogram showed a shortened PR interval (0.10 sec) with normal QRS complexes (Fig. II). On three occasions in hospital he complained of faintness and was found to have atrial flutter with $2: 1$ AV block (Fig. II). Vectorcardiography was normal.

\section{Electrophysiological study}

Fig. I2 illustrates the His bundle electrogram during sinus rhythm with a PR interval of 120 $\mathrm{msec}$, a short P-His interval of $65 \mathrm{msec}$, and a normal His-Q interval. The short $P R$ is therefore due to a reduction in the $\mathrm{P}-\mathrm{H}$ is interval. The $\mathrm{A}$ His interval is reduced to $45 \mathrm{msec}$ (lower limit of normal in our laboratory is $55 \mathrm{msec}$ ) and remains reduced during atrial pacing at a cycle length of $620 \mathrm{msec}$ (Fig. 13). As the cycle length is further reduced there is a progressive increase in the AHis interval. These findings are compatible with an anatomical bypass of the atrioventricular node.

\section{Discussion}

The introduction of a simple and safe electrode catheter technique for the consistent recording of His bundle potentials in man has resulted in extensive investigation of the specialized conduction tissue and has greatly extended the information which can be obtained from a standard 12-lead electrocardiogram. By utilizing this technique we have presented further evidence to suggest that an anatomical bypass of the normal atrioventricular conduction system is responsible for the pre-excitation syndrome and its associated tachycardia.

Cases 1, 2, and 3 are examples of the classical Wolff-Parkinson-White syndrome: all have short PR intervals, delta waves, and wide QRS complexes; the degree of pre-excitation varies from time to time. Cases I and 2 show type A, and Case 3 shows type B conduction. Our observations in these patients are consistent with the hypothesis that ventricular pre-excitation results from a bypass of the main His bundle since they all showed QRS complexes formed by fusion between the normal and premature activation processes.

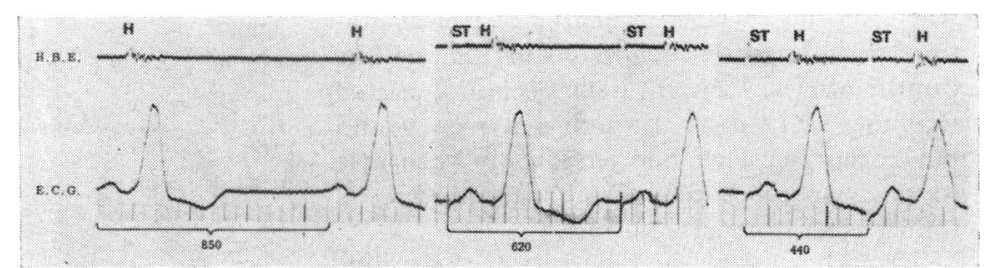

FIG. Io His bundle electrogram of Case 3 during sinus rhythm and during atrial pacing at progressively shorter cycle lengths. A consistent pre-excitation pattern is evident during sinus rhythm and does not significantly alter. The P-His interval progressively increases. The His- $Q$ interval is reduced to $15 \mathrm{msec}$ during sinus rhythm and remains unchanged at shorter cycle lengths.

During sinus rhythm or atrial pacing, when the pre-excitation pattern was seen, the His bundle potential occurred simultaneously with the QRS complex or preceded it by less than I $5 \mathrm{msec}$. As the lower limit of normal for the His- $Q$ interval is $35 \mathrm{msec}$ in sinus rhythm and remains unchanged during atrial pacing, the simultaneous activation of the His bundle and QRS complex implies early activation of the ventricle. In none of our patients were we able to show ventricular activation preceding the onset of the His bundle potential though this has been found in two patients (Castellanos et al., 1970).

As the atrioventricular nodal conduction time became prolonged during atrial pacing with progressively shorter cycle lengths, there

FIG. II Electrocardiogram of Case 4 showing the characteristics of the Lown-GanongLevine syndrome with a short $P R$ interval of $O \cdot 10 \mathrm{sec}$, no delta wave, and a normal $Q R S$ complex. The rhythm strip at the bottom shows atrial flutter with 2:I block.

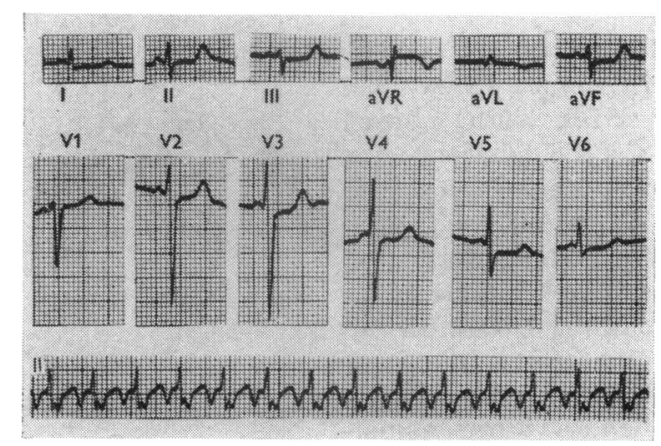


was often an increasing degree of pre-excitation with less conduction occurring via the bundle of His. It might have been anticipated that total activation through a bypass would have occurred if we had technically been able to pace at rates fast enough to produce a totally refractory His bundle.

Though we were unable to study the beginning and the end of the tachycardia produced in Case 2, the most likely, though not the only, explanation for this tachycardia was reentry with antegrade conduction consistently occurring through the His bundle and retrograde conduction bypassing it. Castillo and Castellanos (1970) also showed this during tachycardia produced in 5 cases with WolffParkinson-White syndrome, and we agree with their conclusion that reciprocating tachycardias in this syndrome are probably the result of two separate anatomical communications: antegrade conduction occurring via the normal His bundle and retrograde conduction via the anatomical His bundle bypass. However, re-entry within the atrioventricular node due to nodal dissociation cannot entirely be excluded (Moe and Mendez, 1966).

There is good evidence that in type B conduction the bypass of the bundle of His is right-sided, and circumstantial grounds for believing that it is left-sided in type A (Durrer et al., 1970; Schamroth and Krikler, 1967a). Reciprocating supraventricular tachycardia was induced by right ventricular stimulation in type B, but not in type A (Durrer et al., 1967; Wellens, Schuilenburg, and Durrer, 1971). However, it was only in Case 2 - type A - that we were able to induce paroxysmal tachycardia (by right ventricular stimulation); we are unable to explain this paradox. As the role of extrasystoles in the initiation of arrhythmias in the pre-excitation syndrome has been well substantiated (Durrer et al., 1967), it is possible that the rising incidence of extrasystoles with advancing age may explain the onset or aggravation of arrhythmias late in life: in Case I attacks only started at the age of 62 ; in Case 2 they were relatively mild until the age of 60 .

Case $I$ is of further interest in that he showed two different arrhythmias. Paroxysmal supraventricular tachycardia with normal QRS complexes and loss of the delta wave was probably due to retrograde conduction up the abnormal pathway and antegrade conduction down the bundle of His. Atrial fibrillation also occurred, and could have been due to the impulse returning to the atrial chamber up the normal pathways and finding it out of phase (Schamroth and Krikler, 1967b); antegrade conduction was then down the anomal-

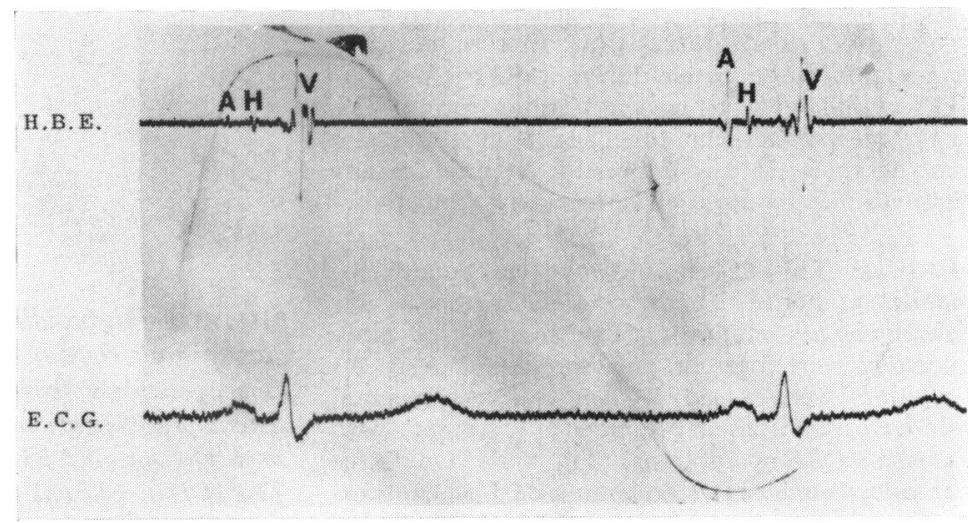

FIG. I2 His bundle electrogram of Case 4 during sinus rhythm with a PR interval of $0.12 \mathrm{sec}$. The $P$-His interval is reduced to 65 msec and the His- $Q$ interval is $55 \mathrm{msec}$. The $A$-His interval is reduced to $45 \mathrm{msec}$ (see text).

ous pathway, with retention of the delta wave. This may be particularly dangerous as there is then a potential for the development of ventricular fibrillation (Dreifus et al., I97I).

The Lown-Ganong-Levine syndrome has also been postulated to be due to an anomalous anatomical structure, the short PR interval representing an AV nodal bypass (Ferrer, 1967; Durrer et al., 1970) as described by James (1963). Recently Castellanos et al. (197I) reported 3 patients in whom His bundle recording showed that during sinus rhythm the reduction in the PR interval was due entirely to a reduction in the A-His interval. In our case, the fourth studied by His

FIG. I3 His bundle electrogram of Case 4 during atrial pacing at progressively shorter cycle lengths. The A-His interval is still reduced to 50 msec at a cycle length of 620 msec but increases to 70 msec at a cycle length of $450 \mathrm{msec}$ and to $90 \mathrm{msec}$ at a cycle length of $390 \mathrm{msec}$ (see text).

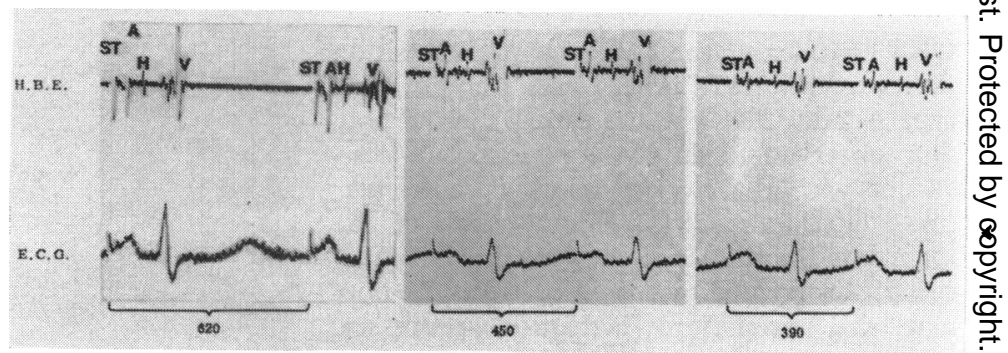


bundle electrograms, we also showed a short A-His interval, representing the time from low right atrial to His bundle activation and normally measuring delay in the AV node. The findings in our case are certainly compatible with a rapidly conducting AV nodal bypass which could possibly be within the AV node itself (Schamroth and Krikler, 1967b). The A-His interval remained short during long cycle lengths but increased as the cycle length shortened. Thus, the normal AV nodal delay was probably not reached until the cycle length was sufficiently short to render the abnormal bypass refractory. Though we did not study it, it is interesting to postulate that the recurrent atrial flutter in this case is initiated by a reciprocating mechanism with antegrade conduction via the AV node and retrograde conduction via an anatomical bypass.

We are grateful to Dr. John Hamer for the vectorcardiogram in Case 4, and to the Editor of the British fournal of Hospital Medicine for permission to reproduce Fig. I and 9 from Krikler (1971).

\section{References}

Castellanos, A., Jr., Castillo, C. A., Agha, A. S., and Tessler, M. (1971). His bundle electrograms in patients with short P-R intervals, narrow QRS complexes, and paroxysmal tachycardias. Circulation, $43,667$.

Castellanos, A., Jr., Chapunoff, E., Castillo, C., Maytin, O., and Lemberg, L. (1970). His bundle electrograms in two cases of Wolff-Parkinson-White (pre-excitation) syndrome. Circulation, 41, 399.

Castillo, C. A., and Castellanos, A., Jr. (1970). His bundle recordings in patients with reciprocating tachycardias and Wolff-Parkinson-White syndrome. Circulation, 42, 271.

Dreifus, L. S., Haiat, R., Watanabe, Y., Arriaga, J., and Reitman, N. (1971). Ventricular fibrillation. A possible mechanism of sudden death in patients with Wolff-Parkinson-White syndrome. Circulation, 43, 520.

Durrer, D., Schoo, L., Schuilenburg, R. M., and Wellens, H. J. J. (1967). The role of premature beats in the initiation and the termination of supraventricular tachycardia in the Wolff-ParkinsonWhite syndrome. Circulation, 36, 644.

Durrer, D., Schuilenburg, R. M., and Wellens, H. J. J. (1970). Pre-excitation revisited. American fournal of Cardiology, 25, 690.

Ferrer, M. I. (1967). New concepts relating to the preexcitation syndrome. Fournal of the American Medical Association, 201, 1038.

James, T. N. (1963). The connecting pathways between the sinus node and AV node and between the right and the left atrium in the human heart. American Heart fournal, 66, 498.

Krikler, D. M. (197I). Disturbances in intracardiac conduction. British fournal of Hospital Medicine, 5,164 .

Lev, M., Leffler, W. B., Langendorf, R., and Pick, A. (1966). Anatomic findings in a case of ventricular pre-excitation (WPW) terminating in complete atrioventricular block. Circulation, 34, 718.

Lown, B., Ganong, W. F., and Levine, S. A. (1952). The syndrome of short P-R interval, normal QRS complex and paroxysmal rapid heart action. Circulation, 5, 693.

Moe, G. K., and Mendez, C. (1966). The physiologic basis of reciprocal rhythm. Progress in Cardiovascular Diseases, 8, 46r.

Rosenbaum, F. F., Hecht, H. H., Wilson, F. N., and Johnston, F. D. (1945). The potential variations of the thorax and the esophagus in anomalous atrioventricular excitation (Wolff-Parkinson-White syndrome). American Heart fournal, 29, $28 \mathrm{I}$.

Schamroth, L., and Krikler, D. M. (1967a). Location of the pre-excitation areas in the Wolff-ParkinsonWhite syndrome. American fournal of Cardiology, 19, 889.

Schamroth, L., and Krikler, D. M. (1967b). The problem of lone atrial fibrillation. South African Medical fournal, 41, 502.

Scherlag, B. J., Lau, S. H., Helfant, R. H., Berkowitz, W. D., Stein, E., and Damato, A. N. (1969). Catheter technique for recording His bundle activity in man. Circulation, 39, 13.

Smithen, C. S., Brown, K., Fluck, D., and Sowton, E. (1972). Analysis of heart block and dysrhythmias by $\mathrm{His}$ bundle electrograms. Cardiovascular $R e-$ search, 6, 129.

Smithen, C. S., and Sowton, E. (197I). Editorial. His bundle electrograms. British Heart fournal, 33, 633.

Wellens, H. J. J., Schuilenburg, R. M., and Durrer, D. (I97I). Electrical stimulation of the heart in patients with Wolff-Parkinson-White syndrome, type A. Circulation, 43, 99.

Requests for reprints to Dr. D. M. Krikler, The Prince of Wales's General Hospital, London NI5 4AW. 